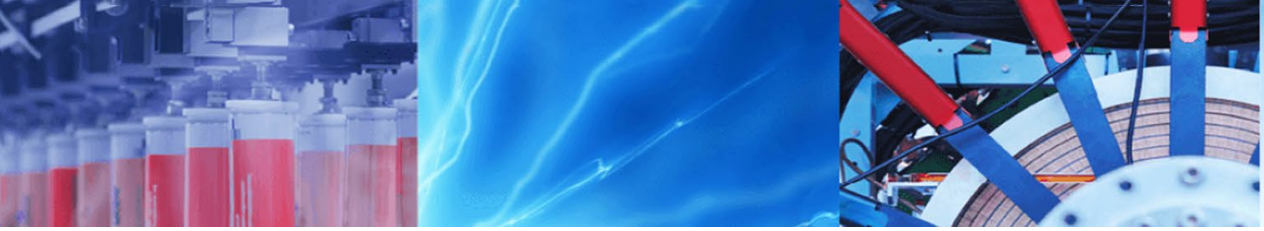

Research Article

\title{
Self-assemblying behaviour of activated microporous carbon microspheres functionalized via water filtration process
}

\author{
Gamze Taylan $^{1} \cdot$ Alparslan Demirural $^{2} \cdot$ Tarik Baykara $^{1}$
}

Received: 8 January 2020 / Accepted: 9 June 2020 / Published online: 16 June 2020

(c) Springer Nature Switzerland AG 2020

\begin{abstract}
Activated carbon spheres are truly innovative materials with their porous micro/nanostructure that gives them tunable and controllable surface properties (i.e. high surface area, size and orientation of pores etc.). They have great potential for applications as adsorbents for cleaning/purification of water, air and chemical substances, catalyst supports, energy storage and conversion, adsorption of chemical warfare agents, filtration and others. Magnetic functionalization of highly porous and high surface areas of activated carbons have received considerable attention for their more sophisticated and advanced utilization as "smart" and "self-assemblying" purposes. In this study, a novel fabrication technique was used to synthesize activated high compressive strength nanoporous carbon spheres. The functionalization via adsorption/ impregnation of magnetic substances was occured during a well-known water treatment-filtering route to fill the pores with nano-sized magnetic particles. Unusually active and dynamic interactions of the magnetic phases were observed upon contacting into each other revealing self-assemblying behaviour of activated carbon microspheres. Dynamic magnetical interaction of self assemblying behaviour of these spheres may have further and advanced applications for variety of purposes.
\end{abstract}

Keywords Spherical activated carbons · Functionalization · Microfiltration · Water treatment · Magnetic interaction · Self-assemblying behaviour

\section{Introduction}

Activated carbon spheres are truly innovative materials that present a variety of unusual applications in various fields including adsorbents for purification of water, air and chemical substances, catalyst supports, energy storage and conversion, adsorption of chemical warfare agents, filtration and others. The most unique property of carbon spheres is attributed to their activated microporous structure that gives them tunable and controllable surface and microstructural properties (i.e. high surface area, size and orientation of pores, high sorption capability etc.). There have been numerous synthesis techniques to obtain porous activated spherical carbons including nanoporous structure through hydrothermal carbonization, emulsion/suspension polymerization and carbonization processes [1-3].

Porous activated carbon microspheres are also being widely used as filtering media in water treatment and remediation for the removal of organic and inorganic pollutants, toxic agents and chemical substances. Such porous activated carbon spheres are being utilized for the cleaning up of oil spills, treatment of waste water i.e., removal of copper ions [4], pollutants [5] and for the removal of chemically toxic warfare agents in the defensive measure against CBRN threats. They have very high surface area (1200-1700 $\mathrm{m}^{2} / \mathrm{g}$ ) along with homogeneously distributed pores of varying sizes and controllable

Tarik Baykara, tbaykara@dogus.edu.tr | ${ }^{1}$ Advanced Mechanical Lab, Mechanical Engineering Department, Dogus University, Kadikoy, Turkey. ${ }^{2}$ Istanbul Aydın University, Kucukcekmece, Istanbul, Turkey. 
structures. Other attractive properties include low density, high porosity, chemical inertness, high sorption capabilities, appropriate recyclability, environmental friendliness, bio-compatibility, ease of manipulation under severe conditions and reasonable costs [4-7]. The functionalization of nano carbon spheres is reported to offer tremendous opportunities with desired properties for various applications in adsorption, energy conversion and storage, biomedical and environmental uses and utilizations [1]. Recently, magnetic functionalization of activated carbons has received considerable attention due to their high surface area and possibility of imposing magnetic properties as a new and non-costly adsorbents [4]. In this regard, magnetically responsive intelligent carbon spheres for oil sorption can be used for a variety of purposes including oil spill clean up, oil recovery, polluted water clean up, water remediation and for other related applications [8-13]. The high affinity between polluting agents and metal oxides/ hydroxides such as iron, titanium, manganese and aluminum in aqueous solutions has been extensively demonstrated. Specifically, due to their high adsorption capacity and low cost, iron hydro(oxides) anchored in the porous carbon structure is widely used for water treatment and remediation. Spherical activated nanoporous carbons are perfect carriers for this purpose due to their high porosities and high surface areas in which iron hydro(oxide) can be anchored inside the structure $[9,10]$. Nanocomposite Fe-based anodes, where the crystalline FeOx nanoparticles are distributed within nanoporous carbon spheres was also reported as high capacity anode for rechargeable alkaline batteries [11].

Due to their extraordinary micro- and/or meso, nanoporous structures, they have been attracting extensive interest for their more sophisticated and advanced utilization as "smart" and "self-assemblying" materials as well [14]. Self-assembly mechanisms have been considered as one of the critical smart/autonomous processes to built up ensembles/building blocks of nano-structures. The concept of "self-assemblying" is attributed to the features of interacting of individual components including shape, geometry and forms, surface properties (like surface area), magnetic properties, mass etc. In the self-assembly of micron-sized structures, it is possible to design, tailor and balance attractions and repulsions through molecular interactions such as magnetic, electromagnetic, gravitational, entropic interactions [14]. Using magnetic interactions leading into self-organization to form/shape desired patterns and functions is the key driver for the applications of self assembly for various purposes including drug delivery, energy conversion, complex smart systems and others. Activated nanoporous carbon spheres can be modified and functionalized to respond certain external stimulations such as electrical, photonic, chemical, thermal and magnetic inputs. Such "smartness" can be achieved through the functionalization of the spherical porous structures directly or indirectly with magnetic substances (such as iron oxide and others). Therefore, routing the materials to the desired locations or autonomously directing the spheres via self assemblying mechanism is quite possible. The anchoring/adsorbing magnetic substances into the carbon structure should be carefully designed in which iron containing magnetical subtances could freely interact onto each other for self assemblying process. For this purpose, a specific drying treatment of impregnated activated carbon surfaces may lead to the loosely anchored or physically adsorbed magnetic substances magnetically interacting with the neighbouring surfaces.

Magnetic properties of carbon-based materials which are metal-free or containing only negligible amounts of metallic impurities are known fact that attract attention and reported elsewhere [15]. Ferromagnetic behaviour of such carbons are demonstrated even above room temperature. Such a behaviour is attributed to the defects or disorders in the structure.

In this study, a novel fabrication technique to synthesize high compressive strength activated porous carbon spheres using a patented suspension polymerization, carbonization and activation process (which is described in the next section) was used. Physical adsorption of magnetic colloidal substances was occured during a water treatment route to fill the pores with nano-sized colloidal organic and inorganic substances including magnetic particles which were the remnants of water treatment substances i.e., coagulants-flocculants (ferrite, magnetite, hematite, ilmenite, iron-containing sulfides and chlorides, iron-containing hydro(oxides) etc.) [16]. During the characterization of these magnetically adsorbed spherical porous activated carbons, unusually active and dynamic interaction of the magnetic phases interacting into each other was observed in microscopical examinations using optical and scanning electron microscopy techniques (Fig. 1 and 2). Optical microscope view of impregnated/adsorbed carbon microspheres magnetically attracted onto a $\mathrm{Nd}-\mathrm{Fe}-\mathrm{B}$ magnet was observed as in Fig. 1. Dynamic magnetical interactions via embedded/adsorbed Fe containing magnetical substances could be a convenient route for self assemblying behaviour of these materials and may have further and advanced applications for other purposes.

\section{Materials and method}

Spherical activated carbons used in this study are synthesized using a patented route of suspension polymerization of divinylbenzene and carbonization processes under nitrogen atmosphere at $450-700^{\circ} \mathrm{C}$. Spherical carbons 
Fig. 1 Optical microscope view of interacting and selfassemblying activated carbon spheres on a NdFeB magnet
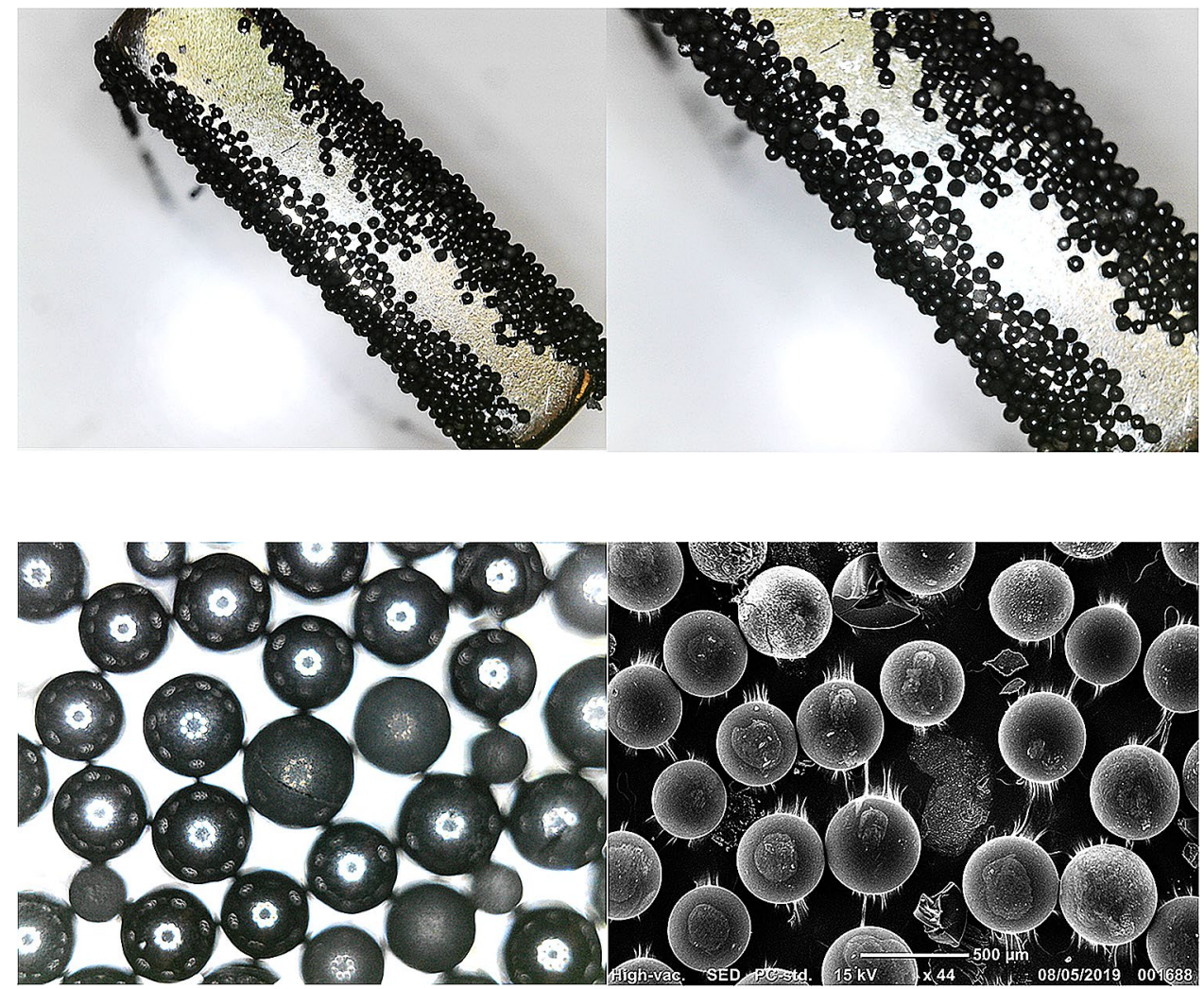

Fig. 2 (left) Optical microscope view of interacting carbon spheres; (right) SEM micrograph of interacting spheres via dynamic material flow onto each other
Table 1 Some physical and chemical properties of the spherical activated carbons [1]

\begin{tabular}{l} 
Physico-chemical properties of spherical activated carbon \\
\hline Size, $0.2-1.4 \mathrm{~mm}$ diameter \\
Surface area $\left(\mathrm{BET} \mathrm{N}_{2}\right), 1160 \mathrm{~m}^{2} / \mathrm{g}$ \\
Mechanical strength under compression, $271-781 \mathrm{~N} / \mathrm{mm}^{2}$ \\
Hardness, $540 \mathrm{~N} / \mathrm{mm}^{2}$ \\
Bulk density, $0.71 \mathrm{~g} / \mathrm{cc}$ \\
lodine number, 785 \\
Ash content, $0.10 \%$ \\
C content, $95.4 \%$ \\
Moisture content, $0.53 \%$ \\
$\mathrm{~V}_{\text {micro, }} 0.478 \mathrm{cc} / \mathrm{g}$ \\
$\mathrm{pH}, 8.05$
\end{tabular}

containing magnetical substances via conventional coagulation-flocculation process with aluminum sulfate (alum), ferric chloride, and ferric sulfate soluton. They were left to dry for a prolonged period of time. Hence, samples with varying sizes (spherical carbon samples with sizes of 200 , 400,600 and $800 \mu \mathrm{m}$ spherical diameters) were obtained which were adsorbed/filled with magnetically active iron

pensions of magnetical particles. Such suspensions were 


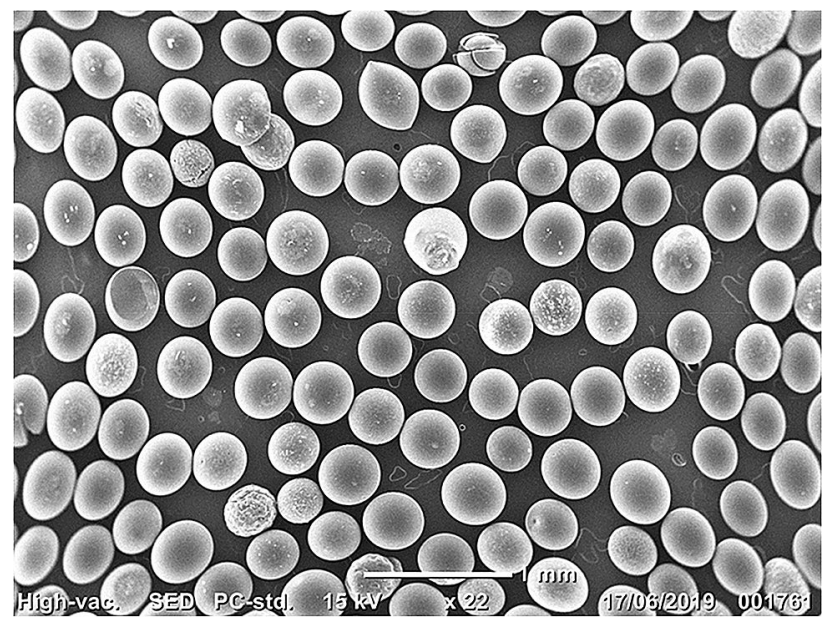

Fig. 3 SEM micrograph of the spherical activated carbons (as received from the activation process; before water filtering/treatment process)

containing magnetic substances. Samples were then characterized for the performance and their pore structures through use of SEM-Scanning Electron Microscopy (SEM) techniques. Microstructural analysis was performed using SEM-Scanning Electron Microscopy and EDS techniques on the surfaces of the spheres. JEOL 6000 Plus desk-top SEM with EDS analysis unit was used. Dried carbon spheres were stored for a prolonged period of time (more than a month) and samples of carbon microspheres were taken from these batches and carefully mounted on a sticky band and flushed with air to remove any dust or accumulated tiny particles before the SEM examinations.

Measurements for the magnetization curves were completed using the Cryogenic Limited PPMS measurement system and the results are given in Fig. 4 for the impregnated spherical carbon samples with sizes of 200, 400, 600 and $800 \mu \mathrm{m}$ spherical diameters. It should be noted that there is no correlation in between the sizes of spherical carbons and the magnetic behaviour.

\section{Experimental results and discussion}

Adsorbed/Infiltrated by magnetically active substances into pores and their distribution can be seen as tiny nanosized and inhomogeneously distributed white dots. The EDS analysis of one of these substances is given in Fig. 5 . Figure 6 (left) shows the surface structure of an individual carbon microsphere.
EDS analysis reveals the peaks of those Fe containing substances along with other water treatment substances such as $\mathrm{Al}, \mathrm{S}, \mathrm{Ca}$ and others.

Figure 6 (right) reveals the activation of the magnetic substances inside the pores and interacting with the neighbouring spheres.

On smaller magnifications ( $\times 44$ and $\times 90$ ), Fig. 7 shows SEM micrographs of interacting activated carbon spheres via dynamic magnetic substances physically adsorbed into the pores via filtering process. Mutual flowing/gushing and splashing onto each other is observed on some surfaces indicating the self-assemblying behaviour due to magnetic interactions.

It was interesting to observe those gushing/splashing of magnetic substances interacting onto each other due to magnetic attractions. It should also be noted that the SEM microstructural examination was conducted under vacuum conditions. In this regard, these in situ magnetic interactions in between the carbon surfaces were indicating a kind of equilibrium state depicting those burst substances interacted onto each other as detailed in Figs. 7 and 8 (right).

The SEM micrographs in Fig. 8 show higher magnifications $(\times 100$ and $\times 270)$ activated spherical carbon spheres, demonstrating detailed magnetic interactions in between the surfaces. A detailed gushing or splash-like flow of the magnetic substances interacting onto each other can be observed in these micrographs. Those magnetic substances are surely nano-sized particles and stayed in constant interaction in between the spheres.

In Fig. 9, an optical micrograph of the activated carbon spheres is shown to demonstrate magnetical interaction in between the surfaces along with self assemblying behaviour. Note that the particles vary in size and they tend to form linear patterns and arrays. Interactions via bridging of surfaces seem to occur in between the closest faces of the spherical surfaces. In some instances, multibridging between varying size carbon spheres can also be observed.

The optical micrographs of some activated carbon spheres adsorbed/filled with magnetic substances, shown in Fig. 10, display the self-assemblying behaviour along certain directions with varying orientations. Arrangement and orientation of activated carbon spheres filled/ adsorbed with magnetic substances are shown and demonstrated arrayed and arranged through the straight lines along certain directions. Such alignement and oriented arrangement could be controlled via remote navigation 

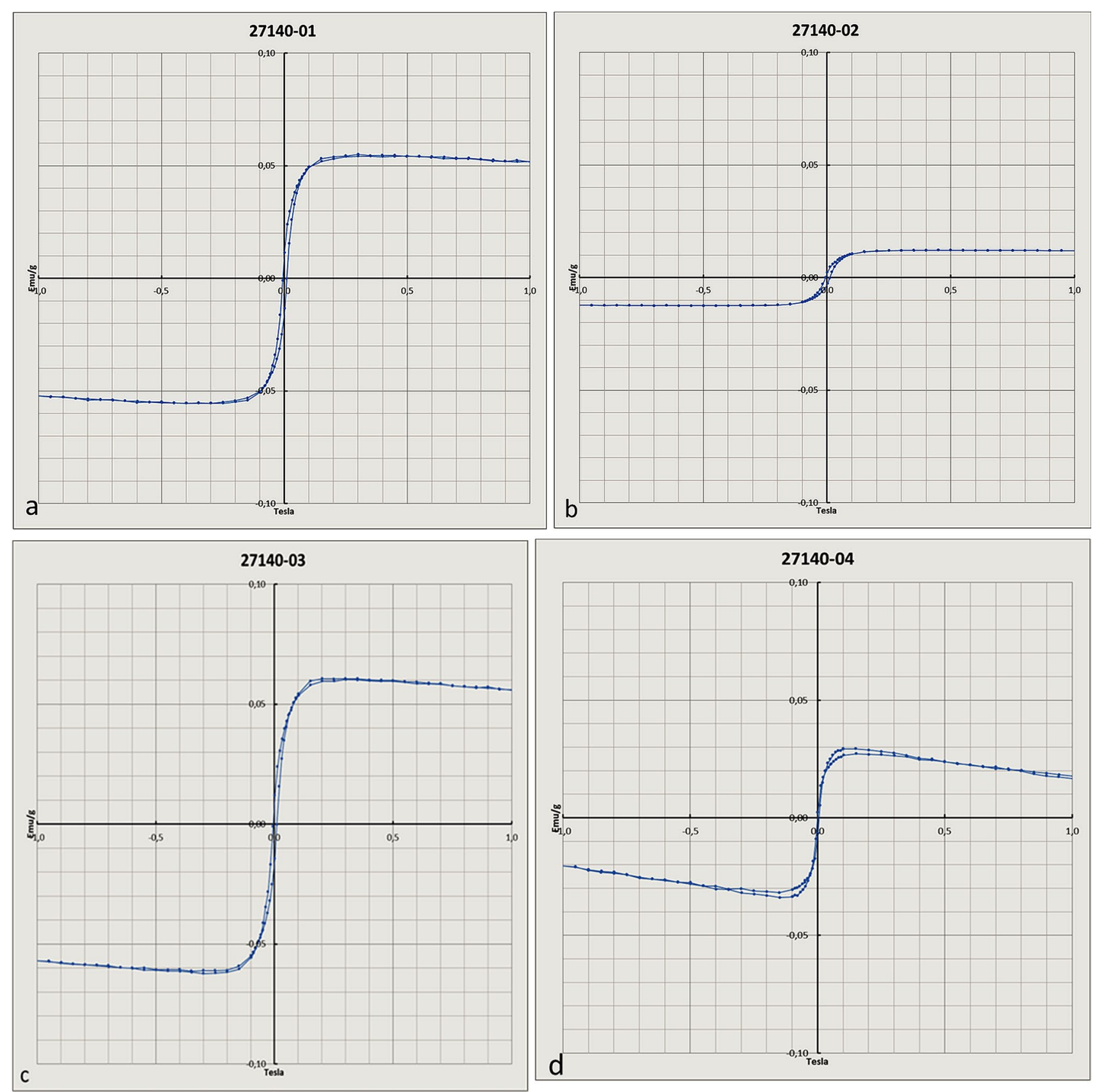

Fig. 4 Magnetization curves of a $200 \mu \mathrm{m} ; \mathbf{b} 400 \mu \mathrm{m} ; \mathbf{c} 600 \mu \mathrm{m}$; d $800 \mu \mathrm{m}$ size samples

using a magnetic source which might be resulted in various applications such as drug delivery and others.

Fractured surfaces of microcarbon spheres are shown in Fig. 11. SEM micrographs were taken just on the fractured edge revealing the porous microstructure along with the magnetic substances adsorbed inside the pores penetrating into the depth of the sphere.
Figure 12 shows a surface of an impregnated porous carbon microsphere. Loosely anchored and/or physically adsorbed white colored magnetic substances are filled the pores and finely distributed throughout the surface. Weak interaction in between the carbon surfaces and iron is a well-known fact, however, porous activated carbons exhibit higher activities due to large surface area and contact between Fe and carbon surfaces [3]. In this figure, 

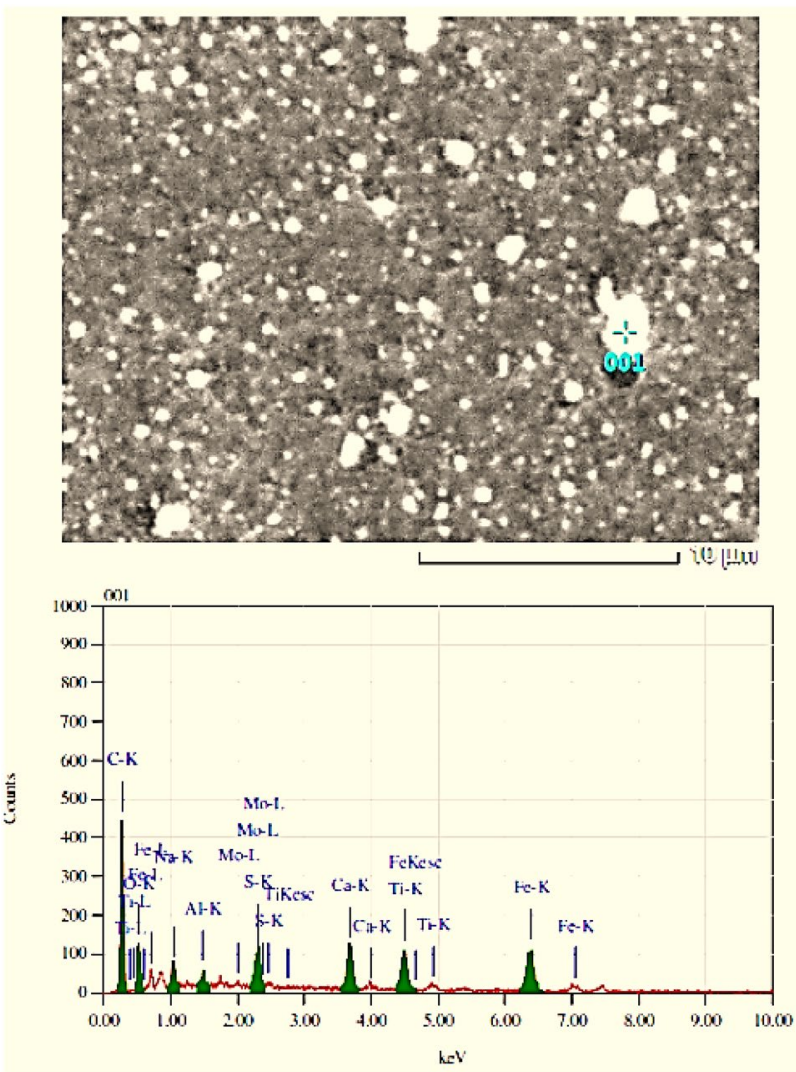

Fig. 5 SEM microstructure and EDS analysis of the filled pore \#001 pore sizes are not homogeneous. The shape and forms of pore sites are randomly filled with varying amounts of magnetic substances. EDS analysis reveals Fe peaks along with a strong $C$ peak and other water treatment/filtering substances (from coagulation and flocculation processes). The results of the further investigations of carbon spheres i.e., those demonstrating gushing/splashing substances into each other due to magnetic interactions is given in Fig. 13. Magnetic interactions via a splash-like burst of magnetic substances from multiple points of a porous surface were seen in this SEM micrograph. The EDS analysis just on the exiting point of a filled pore i.e., the point 001 reveals $\mathrm{C}$ and $\mathrm{Fe}$, $\mathrm{S}$ peaks.

Magnetically filled/adsorbed spherical nanoporous activated carbons, unusually active and dynamically attracting magnetic phases to interact into each other were shown to demonstrate a self-imposed magnetic self-assemblying behaviour. Such extra ordinary behaviours were examined using optical and scanning electron microscopy techniques along with EDS analyisis (Figs. 10, 11,12 and 13). It is known that magnetically functionalized particulates have the potential to lead the required conditions to provide controllable building blocks due to directional magnetic interactions. Magnetically impregnated or embedded particles can be remotely controlled and/ or manipulated via external stimulation $[18,19]$. In-situ dynamic magnetical interactions were observed during
Fig. 6 SEM micrographs: (left) Surface of an individual carbon microsphere adsorbed/filled with magnetical substances; (right) start of the interaction with the neighbouring surfaces
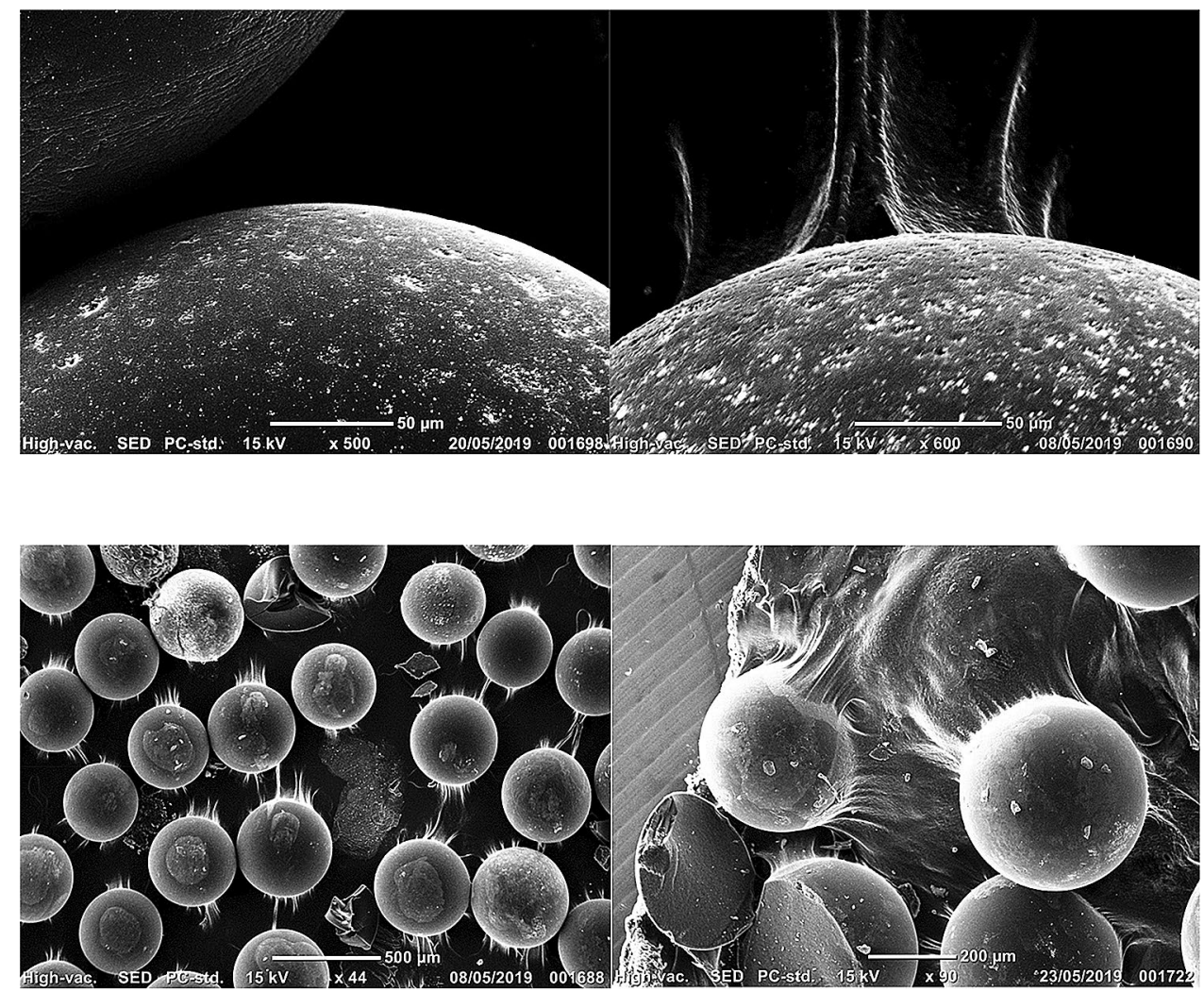

Fig. 7 SEM micrograph of interacting activated carbon spheres via dynamic magnetic material flow onto each other
SN Applied Sciences A SPRINGER NATURE journal 
Fig. 8 Higher magnification of SEM micrographs of interacting activated carbon spheres via dynamic magnetic material flow onto each other

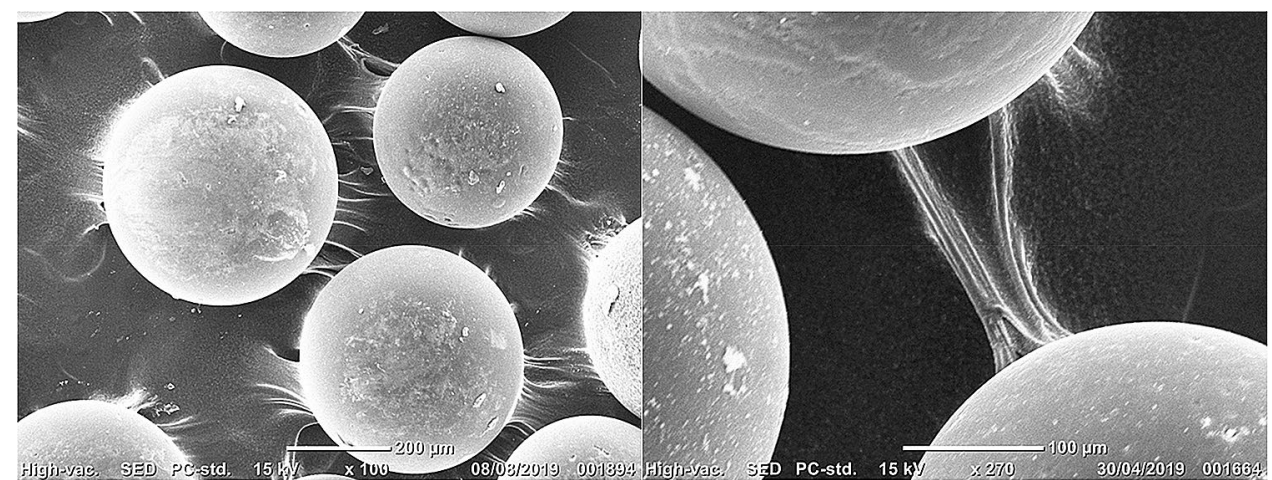

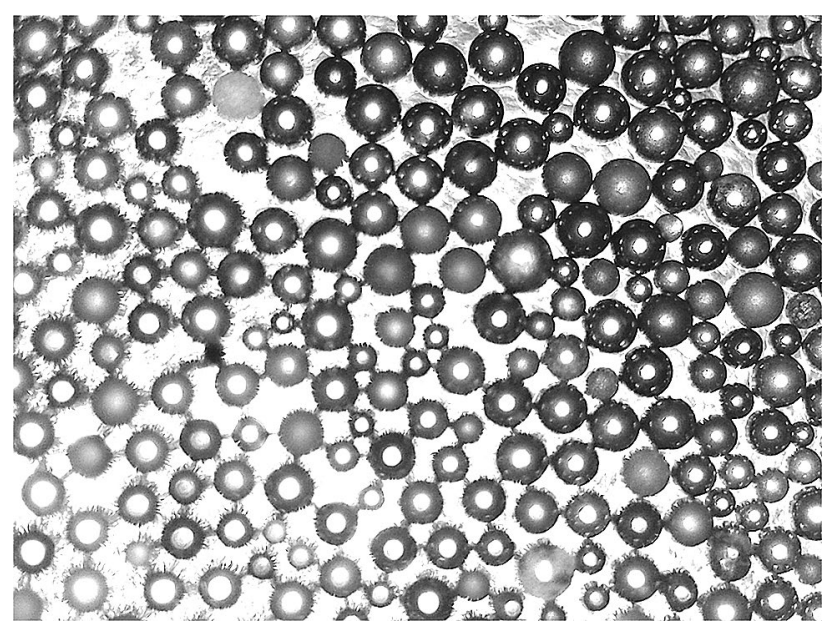

Fig. 9 Optical microscope view of interacting and self-assembled carbon spheres
SEM observation which could be a convenient route for self assemblying behaviour of these materials. Controllable and programmable arrangements via directionality of magnetic interactions may have further and advanced applications for other purposes (such as drug delivery i.e., nano-porous carbon would be a good drug carrier).

\section{Conclusion}

Magnetical adsorption/impregnation of magnetical substances via water treatment-filtering process into the pores of spherical nanoporous activated carbons reveals an unusually active and dynamic interaction of the magnetic phases attracting into each other. Self-imposed magnetic self-assemblying behaviour i.e., arranging and arraying carbon spheres along certain orientations was observed
Fig. 10 Optical microscope view of interacting and selfassemblying activated carbon spheres

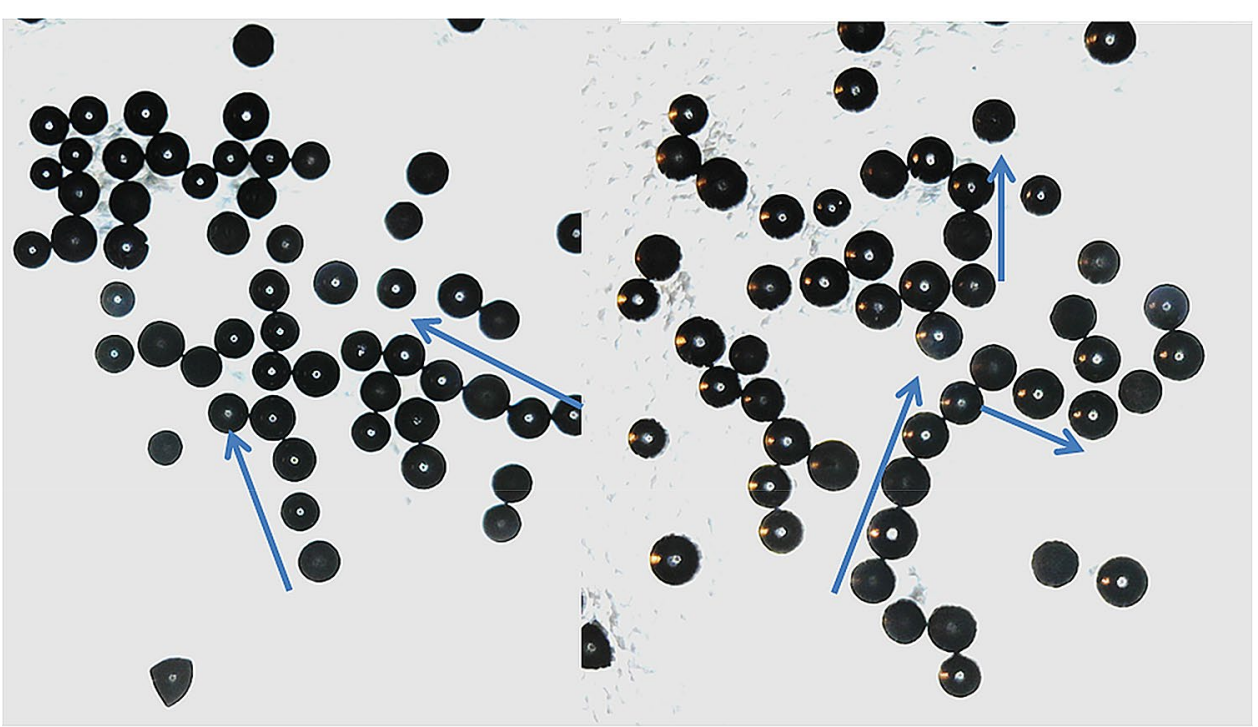


Fig. 11 Fractured surfaces of microcarbon spheres showing the porous structure
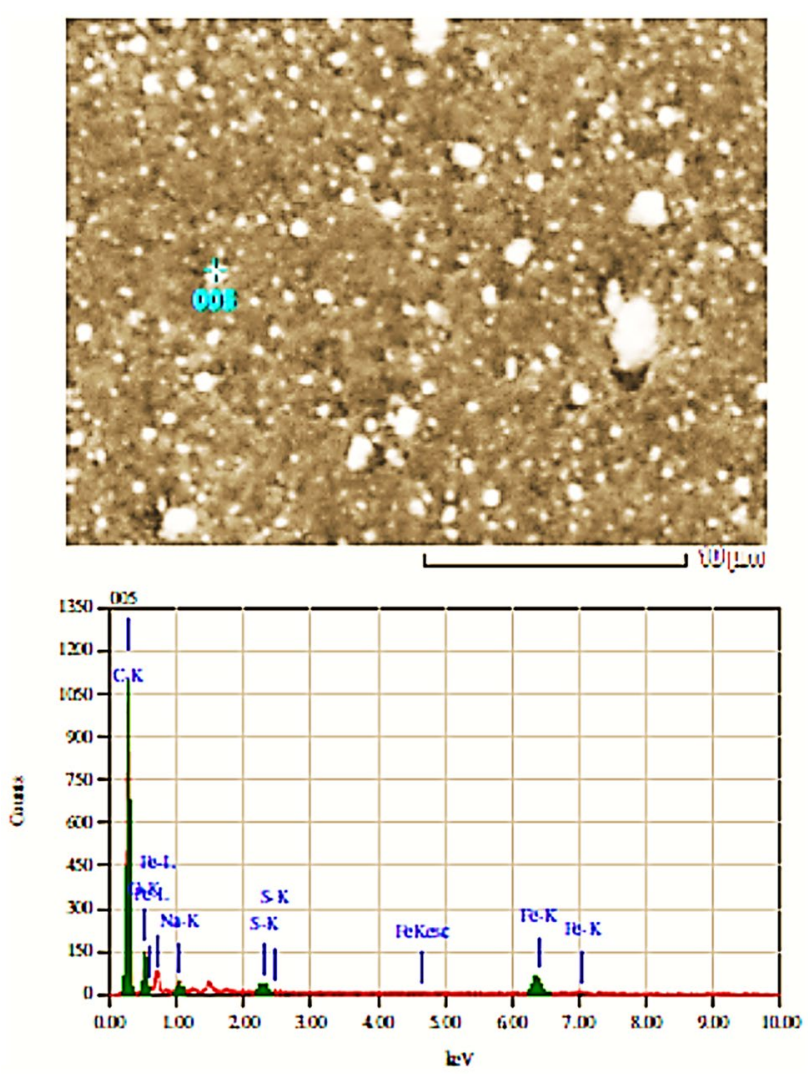

Fig. 12 EDS analysis of the activated carbon spheres at the point $\# 001$
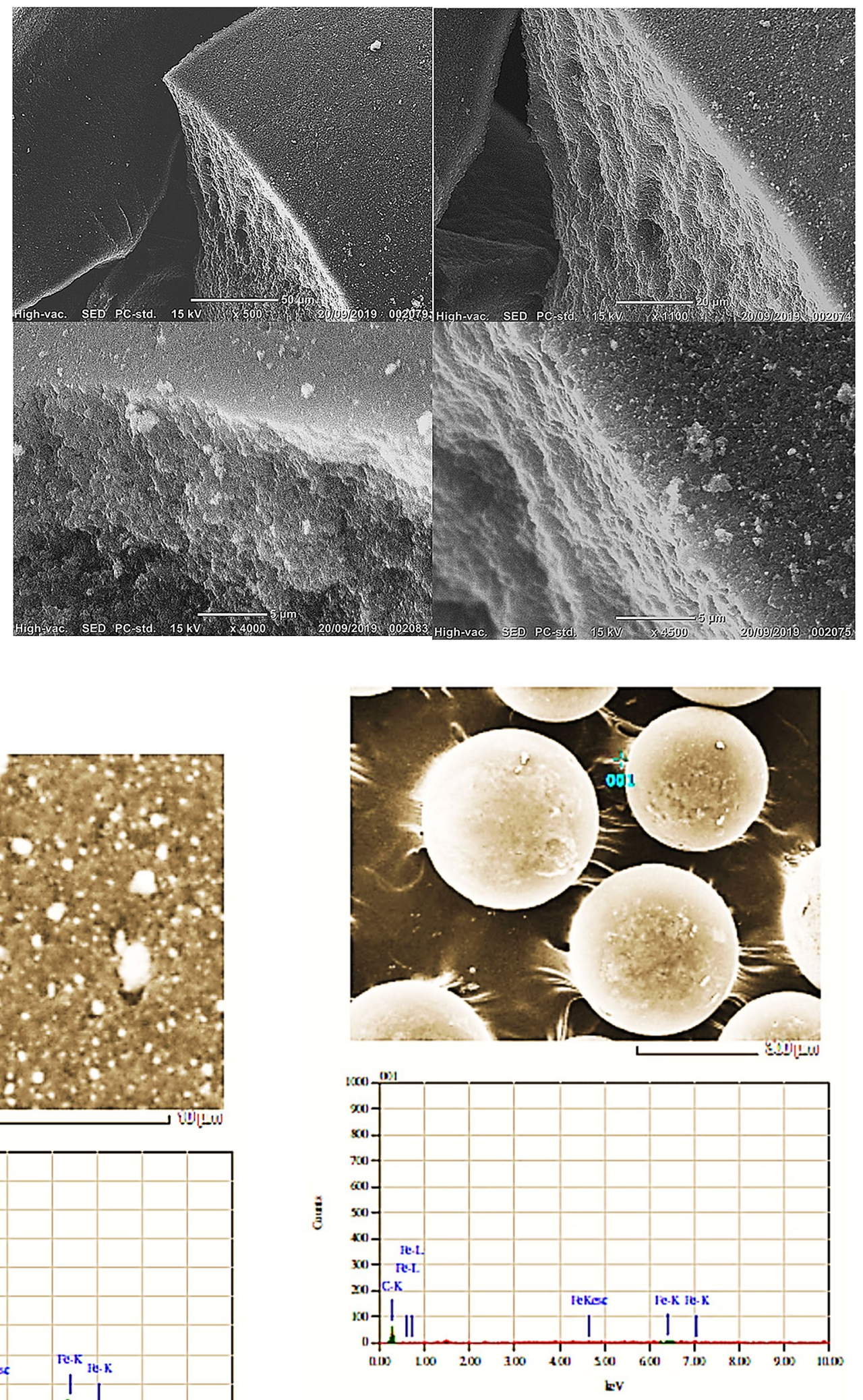

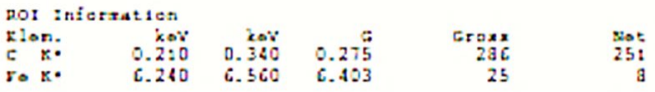

Fig. 13 EDS analysis of the activated carbon spheres at the point $\# 001$ and elemental info indicating $C$ and Fe contents

\section{SN Applied Sciences}


using SEM and optical microscopy techniques along with EDS analyisis to show in situ dynamic magnetical interactions. This could be a convenient route for smart and functional self assemblying behaviour of these materials. Magnetically functionalized activated surfaces of porous carbons could result in non-contact remote control or manipulation via external magnetical fields. They may have further and advanced applications for other purposes (such as drug delivery i.e., nano-porous carbon would be a good drug carrier).

\section{Compliance with ethical standards}

Conflict of interest The author(s) declare that they have no conflict of interest.

\section{References}

1. Yenisoy-Karakas S, Aygun A, Gunes M, Tahtasakal E (2004) Physical and chemical characteristics of polymer-based spherical activated carbon and its ability to adsorb organics. Carbon 42:477-484

2. Liu J, Wickramaratne NP, Qiao SZ, Jaroniec M (2015) Molecularbased design and emerging applications of nanoporous carbon spheres. Nat Mater 14(8):763-774

3. Chen Q, Liu G, Ding S, Sheikh MC, Long D, Yoneyama Y, Tsubaki N (2018) Design of ultra-active iron-based Fischer-Tropsch synthesis catalysts over spherical mesoporous carbon with developed porosity. Chem Eng J 334:714-724

4. Whitesides GM, Grzybowski B (2002) Self-assembly at all scales. Science 295(5564):2418-2421

5. Gu SY, Hsieh CT, Gandomi YA, Yang ZF, Li L, Fu CC, Juang RS (2019) Functionalization of activated carbons with magnetic Iron oxide nanoparticles for removal of copper ions from aqueous solution. J Molecular Liq 277:499-505

6. Xiong H, Moyo M, Motchelaho MA, Jewell LL, Coville NJ (2010) Fischer-Tropsch synthesis over model iron catalysts supported on carbon spheres: the effect of iron precursor, support pretreatment, catalyst preparation method and promoters. Appl Catalysis A General. 388(1-2):168-178
7. Ma J, Liu C, Chen K (2019) Magnetic carbon bubble for pollutants removal. Sep Purif Technol 225:74-79

8. Busch M, Schmidt W, Migunov V, Beckel A, Notthoff $C$ Kompch A, Bergmann U, Winterer M, Atakan B (2014) Effect of preparation of iron-infiltrated activated carbon catalysts on nitrogen oxide conversion at low temperature. Appl Catal B 160:641-650

9. Nieto-Delgado C, Rangel-Mendez JR (2012) Anchorage of iron hydro (oxide) nanoparticles onto activated carbon to remove As (V) from water. Water Res 46:2973-2982

10. Chang Q, Lin W, Ying WC (2010) Preparation of iron-impregnated granular activated carbon for arsenic removal from drinking water. J Hazard Mater 184(1-3):515-522

11. Lei D, Lee DC, Zhao E, Magasinski A, Jung HR, Berdichevsky G, Steingart D, Yushin G (2018) Iron oxide nanoconfined in carbon nanopores as high capacity anode for rechargeable alkaline batteries. Nano Energy. 48:170-179

12. Liu Xuejiao, Wanga Yin (2019) Activated carbon supported nanoscale zero-valent iron composite: aspects of surface structure and composition. Mater Chem Phys 222:369-376

13. Ali N, Zaman H, Bilal M, Nazir MS, Iqbal HM (2019) Environmental perspectives of interfacially active and magnetically recoverable composite materials-a review. Sci Total Environ 670:523-538

14. Wang N, Deng Z (2019) Synthesis of magnetic, durable and superhydrophobic carbon sponges for oil/water separation. Mater Res Bull 115:19-26

15. Lahderenta E, Lashkul AV, Lisunov KG, Zherebtsov DA, Galimov DM, Titkov AN (2013) Irreversible magnetic properties of carbon nanoparticles. In: EPJ web of conference vol 40, p 08008

16. Howe KJ, Hand DW, Crittenden JC, Trussell RR (2012) Principles of water treatment. Wiley, New Jersey

17. Tahtasakal E et al. (2004) Turkish Patent 200201741 A2 High mechanical strength synthetic spherical carbon-in Turkish

18. Rossi L (2019) Magnetic colloids as building blocks for complex structures: preparation and assembly. Book Chapter 1, In: Frontiers of nanoscience, vol 13, pp 1-22

19. Novak EV, Pyanzina ES, Rozhkov DA, Ronti M, Cerdà JJ, Sintes $T$, Sánchez PA, Kantorovich SS (2018) Suspensions of supracolloidal magnetic polymers: self-assembly properties from computer simulations. J Mol Liq 271:631-638

Publisher's Note Springer Nature remains neutral with regard to jurisdictional claims in published maps and institutional affiliations. 